\title{
Metastatic melanoma masquerading as a furuncle
}

\author{
Imran Aslam¹, Jonathan Konopinski², Nasir Aziz ${ }^{1}$
}

\author{
${ }^{I}$ Department of Dermatology, Howard University, Washington D.C, USA, ${ }^{2}$ Department of Pathology, George Washington \\ University, Washington D.C, USA
}

Corresponding author: Dr. Imran Aslam, E-mail: iaslam@neomed.edu

\begin{abstract}
Melanoma metastasizes to the skin in about $10-17 \%$ of patients. Although there are reports of metastatic melanoma masquerading as panniculitis and erysipelas, it is very uncommon for it to present as an inflammatory skin lesion. When malignant melanoma cells invade the superficial dermal lymphatic vessels it can result in erythema, edema and induration of the overlying skin. This presentation can be problematic for clinicians if they do not suspect melanoma and choose not to biopsy the lesion. We report a case of an elderly man with a history of invasive melanoma who presented with a furuncle-like lesion that was found to be in-transit metastatic melanoma.
\end{abstract}

Key words: Melanoma; In-transit metastasis; Furuncle

\section{INTRODUCTION}

Melanoma metastasizes to the skin in about 10-17\% of patients. Although there are reports of metastatic melanoma masquerading as panniculitis and erysipelas, it is very uncommon for it to present as an inflammatory skin lesion. When melanoma cells invade the superficial dermal lymphatic vessels, they can cause erythema, edema and induration of the overlying skin. This presentation can be problematic for the clinician as a melanocytic neoplasm is rarely suspected. We report a case of an elderly man with a history of invasive melanoma who presented with a furuncle-like lesion that was found to be in-transit metastatic melanoma (ITM).

\section{CASE REPORT}

A 66 year-old Caucasian man presented with a 'boil' on his left leg. The spot had been present for about three months but was getting larger and painful 2-3 weeks prior to presentation. The patient denied any drainage, fevers, chills, or night sweats. His past medical history includes five primary melanomas, dysplastic nevi and multiple basal cell carcinomas. Of the five primary melanomas, three sites were invasive on the right chest, mid back and left calf, and the remaining sites were in situ on the left shoulder and left upper back. All of the melanomas had been excised prior to presentation. The invasive $\mathrm{pT} 4 \mathrm{~b}$ melanoma on the right chest was excised in June 2012 with wide local excision (unknown margins but histologically negative), and two sentinel lymph node biopsies (SLNB) taken from the right and left axillae were both negative for melanoma. The concerning spot on his left calf was close to a scar from an excised pT3b melanoma (stage IIB) that was ulcerated and had a depth of $3.8 \mathrm{~mm}$ and 12 mitotic figures. The leg melanoma was excised in August 2013 with a wide local excision (unknown margins but histologically negative), and two SLNB's were also taken from the left groin which were both negative for melanoma.

Examination of the left calf revealed a $2 \mathrm{~cm}$ round, fluctuant, tender, erythematous nodule without a central punctum located $4 \mathrm{~cm}$ from a large atrophic scar corresponding to the site of the prior excision (Fig. 1). There was no lymphadenopathy.

We used a $4 \mathrm{~mm}$ punch biopsy to make an incision and sent tissue for routine histology. The remaining purulent material was drained and cultured. Both gram stain and bacterial culture were negative. Histologic

\footnotetext{
How to cite this article: Aslam I, Konopinski J, Aziz N. Metastatic melanoma masquerading as a furuncle. Our Dermatol Online. $2018 ; 9(1): 434-437$.

Submission: 27.04.2017; Acceptance: 30.06.2017

DOI: 10.7241 /ourd.20174.123
} 
evaluation showed numerous atypical melanocytes that stained positive for $\mathrm{S} 100$ and HMB45, consistent with metastatic melanoma (Figs. 2 and 3).

Based on the diagnosis of in transit metastasis, the patient was upstaged to IIIC melanoma (T3bN2cM0). He was subsequently referred to medical oncology for further work up. Genetic testing of his melanoma revealed a BRAF wild-type, and a full body PET/ CT scan detected bilateral pulmonary nodules, the largest of which was $1.1 \mathrm{~cm}$ located in the left upper lobe. The patient was subsequently started on the Bristol-Myers Squibb Early Patient Access protocol consisting of combination therapy with ipilimumab and novilumab. A restaging CT scan after cycle 4 demonstrated complete resolution of the pulmonary nodules. He was then continued on novilumab monotherapy for a total of 22 cycles. His treatment course was complicated by a variety of events including hypophysitis with adrenal insufficiency, transaminitis, sepsis and a defibrillator malfunction. Despite these setbacks, he was able to complete his immunotherapy regimen. Eighteen months after our discovery of the ITM, he is off treatment and his most recent restaging CT scans were negative.

\section{DISCUSSION}

Melanoma can be highly aggressive and metastasize to many organs including the gastrointestinal tract, liver, spleen, bone and brain. Prognosis is typically poor for patients with metastatic melanoma, but factors such as site of the first metastasis, number of metastatic sites and duration of remission variably affect prognosis [1]. Distant and visceral metastases have a worse prognosis than local soft tissue spread. Local soft tissue metastasis can be further categorized as satellite or in transit. Satellite metastases are located more than $0.05 \mathrm{~mm}$ but less than $20 \mathrm{~mm}$ from the primary lesion. In transit metastases or ITM's are located more than $20 \mathrm{~mm}$ from the primary lesion but not past the local lymph node basin [2]. Hence, our case illustrates an atypical presentation of ITM.

Although this specific presentation has yet to be described, several authors have documented unusual manifestations of ITM's. Shekhel et al described a case of ITM masquerading as lymphangietasis [3]. Similar to our case, the patient had a history of high risk melanoma in his lower extremity, and it was in this same leg where he developed his lymphangiectasis-like

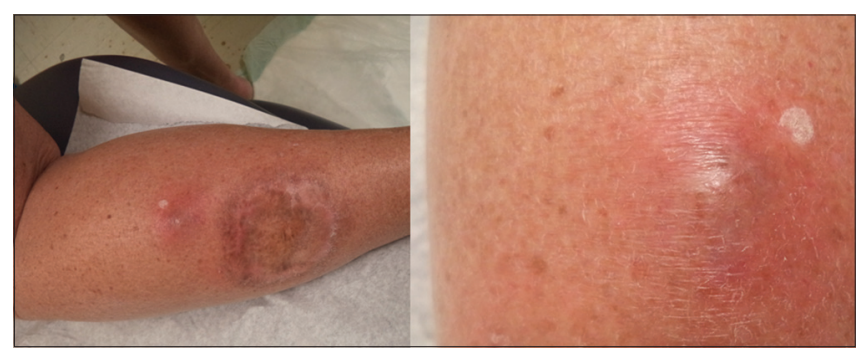

Figure 1: Left lower leg at initial presentation with close up view.

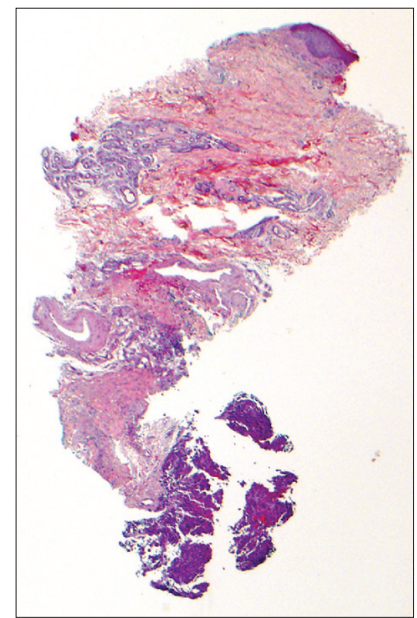

Figure 2: Skin, left lower leg, punch biopsy, low power: A basophilic nodule is seen in the reticular dermis at the base of the biopsy.

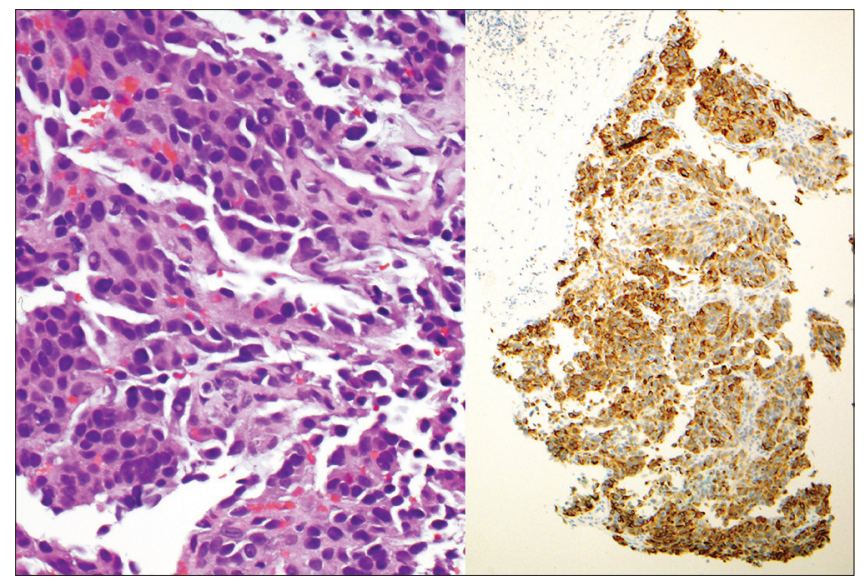

Figure 3: Skin, left lower leg, punch biopsy, immunohistochemistry/ high power: High power: large pleomorphic epithelioid cells with hyperchromatic nuclei and conspicuous intranuclear inclusions. Immunohistochemistry: The atypical epithelioid cells are strongly positive for the melanocytic marker HMB 45.

lesions. While ITM's can occur anywhere on the body, they have been noted to occur more frequently when the primary melanoma involves the lower extremity (19\%) as opposed to other sites such as the head and neck (5\%), trunk (9\%), and upper extremity (8\%) [4]. Also, ITMs on the extremities are associated with a better prognosis than ITMs on other sites [5]. 
Another unique presentation of ITM involved a 73-yearold female with a history of melanoma on her right achilles tendon who presented with a non-fluctuant erythematous plaque on her right anterior leg that clinically appeared to be panniculitis or cellulitis [6]. Once the lesion was biopsied and diagnosed as an ITM, she underwent a lymph node dissection of her groin that was also positive for melanoma. However, subsequent CT and PET scans of her chest, abdomen and pelvis were negative for any additional signs of metastatic disease, and the patient remained clinically stable without any additional symptoms.

Carcinoma erysipeloides or inflammatory carcinoma, is a condition in which an inflammatory response is triggered when malignant cells metastasize to superficial dermal lymphatic vessels resulting in erythematous plaques that resemble erysipelas. Although this phenomenon is most commonly seen in breast cancer, there are a handful of cases in which metastatic melanoma was the culprit [6-10]. One case involved an 87-year-old woman with a history of metastatic melanoma to the axillary lymph node who presented with inflammatory skin changes on her breasts. Her primary site of melanoma was unknown. The patient had tender, indurated, erythematous plaques involving both breasts without any underlying mass. Chest X-ray, mammogram and CT head were all unremarkable. A chest CT revealed enlarged axillary lymph nodes. A skin biopsy confirmed the diagnosis of inflammatory metastatic melanoma. In light of the patient's age and the extent of the area involved, no treatment was done per the family's request. At one month follow up, the patient remained asymptomatic and clinically stable [11].

Importantly, our patient had a negative SLNB at the time of the melanoma diagnosis on his calf. In patients with no clinical signs of nodal disease, the SLNB will detect nodal metastasis in $15-22 \%$ of cases [12]. The rate of ITM or local recurrence in patients with negative SLNB is reported around 4.8\% [13]. While a negative SLNB is a good prognostic indicator, other factors such as primary tumor thickness and ulceration must be considered. Patients with negative SLNB and ulcerated primary tumor or increased Breslow thickness are at higher risk for in transit and distant recurrence as demonstrated in our patient $[12,13]$.

Due to the paucity of case reports involving inflammatory metastatic melanoma, it would be premature to definitively comment on the prognostic implications of this rare presentation. However, it would be reasonable to assume this presentation is likely a poor prognostic indicator [11]. It is known for instance that inflammatory breast carcinoma portends a worse prognosis than other presentations of breast cancer, so inflammatory ITM may also follow similar suit. The few documented cases of inflammatory melanoma have occurred in the setting of fairly advanced disease. However, this could merely reflect the fact that authors are more likely to write case reports about metastatic melanoma rather than cases involving primary cutaneous disease. The prognosis of in-transit metastasis, regardless of inflammation, is considerably worse than local recurrence and is therefore classified as either stage IIIB or IIIC depending on lymph node involvement [14]. The 5 year survival rate for ITM has been reported to be $60.1 \%$ for skin metastasis only and $36.3 \%$ for skin and regional lymph node metastasis [15]. Despite the limitations, this case reinforces the importance of maintaining a strong degree of suspicion of inflammatory lesions located in the vicinity of high risk melanomas. We recommend practitioners to have a low threshold in biopsying such lesions.

\section{REFERENCES}

1. Tas F. Metastatic behavior in melanoma: timing, pattern, survival, and influencing factors. J Oncol. 2012;647684:27.

2. Balch CM, Gershenwald JE, Soong S-j, Thompson JF, Atkins MB, Byrd DR, et al. Final version of 2009 AJCC melanoma staging and classification. J Clin Oncol. 2009;27:6199-206.

3. Shekhel T, Glick RM, Cranmer LD. In-transit metastasis from melanoma presenting as lymphangiectasis: a case report. Cutis. 2009;84:151-8.

4. Calabro A, Singletary SE, Balch CM. Patterns of relapse in 1001 consecutive patients with melanoma nodal metastases. Arch Surg. 1989;124:1051-5.

5. Brady MS, Brown K, Patel A, Fisher C, Marx W. A phase II trial of isolated limb infusion with melphalan and dactinomycin for regional melanoma and soft tissue sarcoma of the extremity. Ann Surg Oncol. 2006;13:1123-9.

6. Cotton J, Armstrong DJ, Wedig R, Hood AF. Melanoma-in-transit presenting as panniculitis. J Am Acad Dermat. 1998;39:876-8.

7. Schneider S, Korting GW. [Erysipelas melanomatosum]. Med Welt. 1975;26:2217-8.

8. Klimpel M. [Atypical erysipelas melanomatosum]. Z Hautkr. 1982;57:783-8.

9. Haupt HM, Hood AF, Cohen MH. Inflammatory melanoma. J Am Acad Dermatol. 1984;10:52-5.

10. Tan BB, Marsden JR, Sanders DS. Melanoma erysipeloides: inflammatory metastatic melanoma of the skin. Br J Dermatol. 1993;129:327-9.

11. Florez A, Sanchez-Aguilar D, Peteiro C, Penaranda JM, Toribio J. Inflammatory metastatic melanoma. J Cutan Pathol. 1999;26(2):105-108.

12. Egger ME, Bhutiani N, Farmer RW, Stromberg AJ, Martin RC 
www.odermatol.com

$2^{\text {nd }}$, Quillo AR, et al. Prognostic factors in melanoma patients with tumor-negative sentinel lymph nodes. Surgery. 2016;159:1412-21.

13. Rutkowski P, Nowecki ZI, Zurawski Z, Dziewirski W, NasierowskaGuttmejer A, Switaj T, et al. In transit/local recurrences in melanoma patients after sentinel node biopsy and therapeutic lymph node dissection. Eur J Cancer. 2006;42:159-64.

14. Grotz TE, Mansfield AS, Erickson LA, Otley CC, Markovic SN, Jakub JW. In-transit melanoma: an individualized approach. Oncology. 2011;25:1340
15. Read RL, Haydu L, Saw RP, Quinn MJ, Shannon K, Spillane, et al. In-transit melanoma metastases: incidence, prognosis, and the role of lymphadenectomy. Ann Surg Oncol. 2015;22:475-481.

Copyright by Imran Aslam, et al. This is an open-access article distributed under the terms of the Creative Commons Attribution License, which permits unrestricted use, distribution, and reproduction in any medium, provided the original author and source are credited.

Source of Support: Nil, Conflict of Interest: None declared. 